Research Article

\title{
Single-Machine Scheduling with Workload-Dependent Maintenance Duration to Minimize Maximum Lateness
}

\author{
Ting Wang and Dehua Xu \\ College of Science, East China Institute of Technology, Nanchang, Jiangxi 330013, China \\ Correspondence should be addressed to Dehua Xu; dhxu@ecit.cn
}

Received 31 August 2014; Accepted 15 September 2014

Academic Editor: Chin-Chia Wu

Copyright (C) 2015 T. Wang and D. Xu. This is an open access article distributed under the Creative Commons Attribution License, which permits unrestricted use, distribution, and reproduction in any medium, provided the original work is properly cited.

A single-machine scheduling problem with a mandatory maintenance whose duration is workload-dependent is considered. The start time of the maintenance is restricted to a time window. The objective is to determine the start time of the maintenance and schedule all the jobs to the machine such that the maximum lateness is minimized. An approximation algorithm based on the classical Earliest Due Date first rule is proposed. It is showed that the proposed algorithm is optimal for some special cases and that it has a tight bound for the scheduling problem under consideration.

\section{Introduction}

Scheduling with due-date related criteria is an important issue in operations management. As is usually the case, if a job cannot be finished before the promised due date, there is a penalty for the producer. When this is the case, the credit standing of the producer also decreases at the same time. As a result, some costumers may turn to another producer in the future. Assume that there is a lateness tolerance for all the costumers. In order to avoid losing too many costumers, minimizing maximum lateness, which measures the worst violation of the due dates, is usually selected as the objective in production scheduling.

To the best of our knowledge, Jackson [1] is the first researcher that considers the maximum lateness objective in the field of scheduling. He shows that the Earliest Due Date first rule, which sequences the jobs in nondecreasing order of their due dates, is optimal for the maximum lateness objective in classical single-machine setting. Since then, numerous works have been done on the same objective for various machine settings and job parameters. For related papers published in recent years, see, for example, [2-7].

Note that a machine may be unavailable sometimes in reality due to various reasons such as refueling, tool changing, and regular check. Taking this factor into consideration, one has to make a coordination between job processing and machine maintenance. In this paper, instead of taking the duration of a maintenance as a constant [8-12] or as a function of its start time [13-15], we assume that the duration of the maintenance is an nonnegative increasing function of its workload before the maintenance [16]. This maintenance duration model is more practical than the start time related model for the fact that the machine may not be always busy before the maintenance in some scenarios. In order to be more realistic, we further assume that the maintenance must be started within a time window.

Formally, the problem under consideration can be described as follows. There are $n$ independent nonpreemptive jobs $J_{1}, J_{2}, \ldots, J_{n}$ to be scheduled on a single-machine with a mandatory maintenance. All jobs are available at time zero. The processing time and due-date of job $J_{j}$ are $p_{j}$ and $d_{j}$, respectively, where $j=1,2, \ldots, n$. The duration of the maintenance is a nonnegative increasing function $f$ of the workload that is scheduled to be carried by the machine before the maintenance, which must be started within time window $[u, v]$, where $0 \leq u \leq v$. The lateness of a job in a schedule is defined as its completion time in the schedule minus its due-date. The objective of the problem is to determine the start time of the maintenance and schedule all the jobs to the machine such that the maximum lateness is minimized.

The rest of this paper is organized as follows. In the next section, we show that the problem under consideration is 
NP-hard. Then, in Section 3, we propose an approximation algorithm with a worst-case analysis. Finally, in Section 4, we conclude the paper.

\section{Computational Complexity}

It is well-known that corresponding classical version of our problem, that is, $1 \| L_{\max }$, can be solved optimally in polynomial time by the Earliest Due Date first rule [17]. However, as can be seen later, our problem is NP-hard.

Theorem 1. The scheduling problem under consideration is NP-hard.

Proof. Consider the restricted version of our scheduling problem where $f$ is a constant function greater than zero, $u=v>0$, and $d_{j}=0$ for every $j \in\{1,2, \ldots, n\}$. It is easy to see that the decision version of the restricted version of our scheduling problem is in NP. Note that the Partition Problem $[17,18]$ which is NP-complete is reducible to the decision version of the restricted version of our scheduling problem. Hence, the decision version of the restricted version of our scheduling problem is also NP-complete. This implies that the restricted version of our scheduling problem is NP-hard. And thus, our scheduling problem (i.e., the nonrestricted version) is NP-hard.

\section{An Approximation Algorithm and Its Worst-Case Analysis}

Note that the scheduling problem under consideration is NP-hard. So, there does not exist any polynomial time optimal algorithm for it unless $\mathrm{P}=\mathrm{NP}$. In what follows, we will present an approximation algorithm named EDDMW (Earliest Due Date first with Maximum Workload before the maintenance) based on the classical Earliest Due Date first rule. The underlying idea of the algorithm is to sort all the jobs according to nondecreasing order of their due dates first and then process as many jobs in this order as possible before maintenance.

Algorithm EDDMW. Consider the following.

Step 1. Sort all the jobs such that $d_{j_{1}} \leq d_{j_{2}} \leq \cdots \leq d_{j_{n}}$, where $\left(j_{1}, j_{2}, \ldots, j_{n}\right)$ is a permutation of $(1,2, \ldots, n)$.

Step 2. Let $n^{\prime}=\max \left\{k \mid \sum_{i=1}^{k} p_{j_{i}} \leq v\right.$ and $\left.k \leq n\right\}$ and $\lambda^{\diamond}=$ $\sum_{i=1}^{n^{\prime}} p_{j_{i}}$

Step 3. If $n^{\prime} \geq 1$, then process the first $n^{\prime}$ jobs in the sorted order consecutively from time zero. If $\lambda^{\diamond}<u$, then keep the machine idle for a time of $u-\lambda^{\diamond}$. Conduct the maintenance from time $\max \left\{u, \lambda^{\diamond}\right\}$ for a duration of $f\left(\lambda^{\diamond}\right)$. If $n^{\prime}<n$, then process the rest of the jobs in the sorted order consecutively from time $\max \left\{u, \lambda^{\diamond}\right\}+f\left(\lambda^{\diamond}\right)$.

Note that Step 1 takes $O(n \log n)$ time while Step 2 takes $O(n)$ time. Assume that the duration function $f(x)$ is given by an oracle which for any given $x$ returns $f(x)$. Then, Step 3 also takes $O(n)$ time. And hence, the running time of the algorithm EDDMW is $O(n \log n)$.

The following notations will be used throughout this paper:

$S^{\diamond}$ : the schedule produced by algorithm EDDMW for the scheduling problem under consideration;

$\lambda^{\diamond}$ : the workload (i.e., the total processing time of the jobs scheduled to be processed) before the maintenance in $S^{\diamond}$;

$L_{j}^{\diamond}$ : the lateness of job $J_{j}$ in schedule $S^{\diamond}$;

$L_{\text {max }}^{\diamond}$ : the maximum lateness of the jobs in schedule

$S^{\diamond}$; that is, $L_{\max }^{\diamond}=\max _{i=1,2, \ldots, n}\left\{L_{j_{i}}^{\diamond}\right\}$;

$S^{*}$ : an optimal schedule for the scheduling problem under consideration;

$\lambda^{*}$ : the workload before the maintenance in $S^{*}$;

$L_{j}^{*}$ : the lateness of job $J_{j}$ in schedule $S^{*}$;

$L_{\max }^{*}$ : the maximum lateness of the jobs in schedule $S^{*}$; that is, $L_{\text {max }}^{*}=\max _{j=1,2, \ldots, n}\left\{L_{j}^{*}\right\}$;

$J_{j_{i} \diamond}$ : the first job that achieves the maximum lateness in $S^{\diamond}$; that is, $i^{\diamond}=\min \left\{k \mid L_{j_{k}}^{\diamond}=\max _{i=1,2, \ldots, n}\left\{L_{j_{i}}^{\diamond}\right\}\right.$;

$J_{j_{l}}$ : the last finished job among the jobs $J_{j_{1}}, \ldots, J_{j_{i} \diamond}$ in $S^{*}$.

Theorem 2. If $n^{\prime}=n$, then $S^{\diamond}$ is optimal.

Proof. If $n^{\prime}=n$, then all the jobs are scheduled to be processed before the maintenance in nondecreasing order of their due date in $S^{\diamond}$, which means that our problem reduces to its classical version without maintenance. Note that Earliest Due Date first rule is optimal for the latter [17]. Therefore, $S^{\diamond}$ is optimal.

Theorem 3. If job $J_{j_{i} \diamond}$ is scheduled to be processed before the maintenance in $S^{\diamond}$, then $S^{\diamond}$ is optimal.

Proof. It is easy to see that

$$
L_{\max }^{\diamond}=L_{j_{i} \diamond}^{\diamond}=\sum_{i=1}^{i^{\diamond}} p_{j_{i}}-d_{j_{i} \diamond} .
$$

Now, consider the following two cases.

Case $1 . J_{j_{l}}$ is scheduled to be processed before the maintenance in $S^{*}$.

It is easy to see that

$$
L_{\max }^{*} \geq L_{j_{l}}^{*} \geq \sum_{i=1}^{i^{\diamond}} p_{j_{i}}-d_{j_{l}} .
$$

Note that $d_{j_{l}} \leq d_{j_{i} \diamond}$. So we have

$$
L_{\max }^{*} \geq L_{j_{l}}^{*} \geq \sum_{i=1}^{i^{\diamond}} p_{j_{i}}-d_{j_{i} \diamond} .
$$


Combining this with (1), we have

$$
L_{\max }^{*} \geq L_{\max }^{\diamond}
$$

which implies that $S^{\diamond}$ is also optimal.

Case 2. $J_{j_{l}}$ is scheduled to be processed after the maintenance in $S^{*}$.

It is easy to see that

$$
L_{\max }^{*} \geq L_{j_{l}}^{*} \geq \sum_{i=1}^{i^{\diamond}} p_{j_{i}}+f\left(\lambda^{*}\right)-d_{j_{l}} .
$$

Note that $f$ is nonnegative and that $d_{j_{l}} \leq d_{j_{i} \triangleright}$. So we have

$$
L_{\max }^{*} \geq L_{j_{l}}^{*} \geq \sum_{i=1}^{i^{\diamond}} p_{j_{i}}-d_{j_{i} \diamond} .
$$

Combining this with (1), we have

$$
L_{\max }^{*} \geq L_{\max }^{\diamond}
$$

which implies that $S^{\diamond}$ is also optimal.

This completes the proof.

Now, let $g(x)=f(x)-x, g_{\max }=\max \{g(x) \mid 0 \leq x \leq u\}$, and $g_{\min }=\min \{g(x) \mid 0 \leq x \leq u\}$.

Theorem 4. If job $J_{j_{i} \diamond}$ is scheduled to be processed after the maintenance in $S^{\diamond}$, then

$$
L_{\max }^{\diamond} \leq L_{\max }^{*}+ \begin{cases}g_{\max }-g_{\min } & \text { if } \lambda^{\diamond} \leq u, \lambda^{*} \leq u \\ g_{\max }-g(u) & \text { if } \lambda^{\diamond} \leq u, \lambda^{*}>u \\ f(v)-u-g_{\min } & \text { if } \lambda^{\diamond}>u, \lambda^{*} \leq u \\ f(v)-f(u) & \text { if } \lambda^{\diamond}>u, \lambda^{*}>u\end{cases}
$$

and each of the bounds is tight.

Proof. We declare that job $J_{j_{l}}$ cannot be scheduled to be processed before the maintenance in $S^{*}$. To see this is the case, if $J_{j_{l}}$ is scheduled to be processed before the maintenance in $S^{*}$, then by the definition of $J_{j_{l}}$, jobs $J_{j_{1}}, \ldots, J_{j_{i} \diamond}$ must be scheduled to be processed before the maintenance too, which clearly contradicts the assumption of the theorem. So, in what follows, we assume that job $J_{j_{l}}$ is scheduled to be processed after the maintenance in $S^{*}$.

Now, consider the following four cases.

Case 1. $\lambda^{\diamond} \leq u$ and $\lambda^{*} \leq u$. It is easy to see that

$$
L_{\max }^{\diamond}=L_{j_{i} \diamond}^{\diamond}=\sum_{i=1}^{i^{\diamond}} p_{j_{i}}+\left(u-\lambda^{\diamond}\right)+f\left(\lambda^{\diamond}\right)-d_{j_{i} \diamond}
$$

and that

$$
L_{\max }^{*} \geq L_{j_{l}}^{*}=\sum_{i=1}^{i^{\diamond}} p_{j_{i}}+\left(u-\lambda^{*}\right)+f\left(\lambda^{*}\right)-d_{j_{l}} .
$$

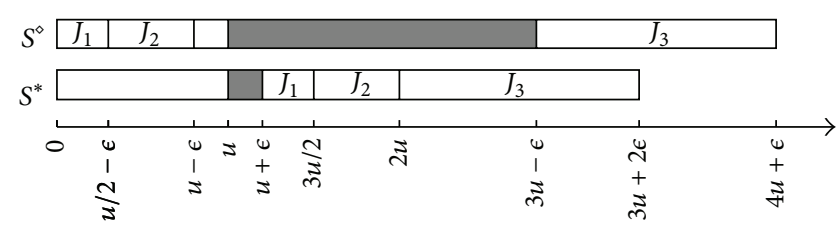

FIGURE 1: Schedules for Instance 1.

Combining (9) and (10) with the definitions of $g_{\max }$ and $g_{\min }$, respectively, we have

$$
L_{\max }^{\diamond}=L_{j_{i} \diamond}^{\diamond} \leq \sum_{i=1}^{i^{\diamond}} p_{j_{i}}+u+g_{\max }-d_{j_{i} \diamond}
$$

and that

$$
L_{\max }^{*} \geq L_{j_{l}}^{*} \geq \sum_{i=1}^{i^{\diamond}} p_{j_{i}}+u+g_{\min }-d_{j_{l}}
$$

Recall that $d_{j_{l}} \leq d_{j_{i} \diamond}$. Combining this with (12), we have

$$
L_{\max }^{*} \geq L_{j_{l}}^{*} \geq \sum_{i=1}^{i^{\diamond}} p_{j_{i}}+u+g_{\min }-d_{j_{i} \diamond} .
$$

Combining (13) with (11), we have

$$
L_{\max }^{\diamond} \leq L_{\max }^{*}+g_{\max }-g_{\text {min }} .
$$

To see that this bound is tight, consider the following instance.

Instance 1. Consider $n=3, p_{1}=u / 2-\epsilon, p_{2}=u / 2, p_{3}=$ $v-u+2 \epsilon, d_{1}=u / 3, d_{2}=u / 2, d_{3}=u, f(x)=2 x+\epsilon$, and $v=2 u$, where $\epsilon \leq u / 4$.

It can be checked that for this instance $L_{\text {max }}^{\diamond}=L_{3}^{\diamond}=3 u+\epsilon$ while $L_{\max }^{*}=L_{3}^{*}=2 u+2 \epsilon$ (see Figure 1). Note that for this instance $g(x)=f(x)-x=x, g_{\max }=u$, and $g_{\min }=0$. So we have $L_{\text {max }}^{\diamond} \rightarrow L_{\text {max }}^{*}+g_{\text {max }}-g_{\text {min }}$ as $\epsilon \rightarrow 0$ for Instance 1 .

Case 2. $\lambda^{\diamond} \leq u$ and $\lambda^{*}>u$. It is easy to see that in this case we have (11) and

$$
L_{\max }^{*} \geq L_{j_{l}}^{*}=\sum_{i=1}^{i^{\diamond}} p_{j_{i}}+f\left(\lambda^{*}\right)-d_{j_{l}} .
$$

Recall that $d_{j_{l}} \leq d_{j_{i} \diamond}$. Combining this with (15), we have

$$
L_{\max }^{*} \geq L_{j_{l}}^{*}=\sum_{i=1}^{i^{\diamond}} p_{j_{i}}+f\left(\lambda^{*}\right)-d_{j_{i} \diamond} .
$$

Note that $\lambda^{*}>u$ and that $f$ is increasing. So we have

$$
L_{\max }^{*} \geq L_{j_{l}}^{*} \geq \sum_{i=1}^{i^{\diamond}} p_{j_{i}}+f(u)-d_{j_{i} \diamond} .
$$

Combining this with (11), we have

$$
L_{\max }^{\diamond} \leq L_{\max }^{*}+u+g_{\max }-f(u) .
$$




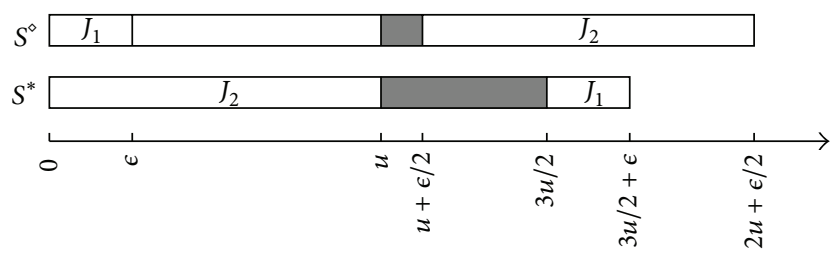

Figure 2: Schedules for Instance 2.

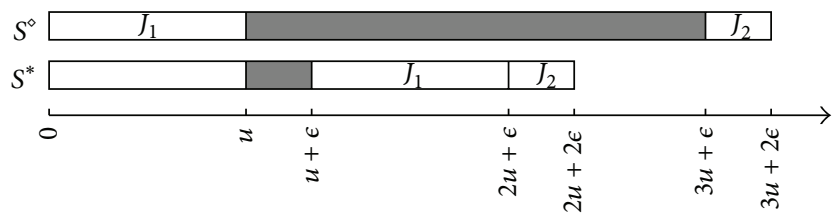

Figure 3: Schedules for Instance 3.

Note that $g(x)=f(x)-x$. So we have

$$
L_{\max }^{\diamond} \leq L_{\max }^{*}+g_{\max }-g(u) .
$$

To see that this bound is tight, consider the following instance.

Instance 2. Consider $n=2, p_{1}=\epsilon, p_{2}=u, d_{2}=d_{1}+\epsilon / 2$, $f(x)=x / 2$, and $v=u$, where $\epsilon \leq u / 4$.

It can be checked that for this instance $L_{\max }^{\diamond}=L_{2}^{\diamond}=2 u-$ $d_{1}$ while $L_{\max }^{*}=L_{1}^{*}=3 u / 2+\epsilon-d_{1}$ (see Figure 2). Note that for this instance $g(x)=f(x)-x=-x / 2, g_{\max }=0$, and $g(u)=-u / 2$. So we have $L_{\max }^{\diamond} \rightarrow L_{\max }^{*}+g_{\max }-g(u)$ as $\epsilon \rightarrow 0$ for Instance 2 .

Case 3. $\lambda^{\diamond}>u$ and $\lambda^{*} \leq u$. It is easy to see that in this case we have (13) and

$$
L_{\max }^{\diamond}=L_{j_{i} \diamond}^{\diamond}=\sum_{i=1}^{i^{\diamond}} p_{j_{i}}+f\left(\lambda^{\diamond}\right)-d_{j_{i} \diamond} .
$$

Note that $\lambda^{\diamond} \leq v$. So, by (20), we have

$$
L_{\max }^{\diamond}=L_{j_{i} \diamond}^{\diamond} \leq \sum_{i=1}^{i^{\diamond}} p_{j_{i}}+f(v)-d_{j_{i} \diamond} .
$$

Combining this with (13), we have

$$
L_{\max }^{\diamond} \leq L_{\max }^{*}+f(v)-u-g_{\min } .
$$

To see that this bound is tight, consider the following instance.

Instance 3. Consider $n=2, p_{1}=v, p_{2}=\epsilon, d_{2}=d_{1}+\epsilon / 2$, $f(x)=2 x+\epsilon$, and $v=u$, where $\epsilon \leq u / 4$.

It can be checked that for this instance $L_{\max }^{\diamond}=L_{2}^{\diamond}=3 u+$ $3 \epsilon / 2-d_{1}$ while $L_{\max }^{*}=L_{2}^{*}=2 u+3 \epsilon / 2-d_{1}$ (see Figure 3$)$. Note that for this instance $g(x)=f(x)-x=x+\epsilon, g_{\min }=\epsilon$, and $f(v)=2 u+\epsilon$. So we have $L_{\max }^{\diamond} \rightarrow L_{\max }^{*}+f(v)-u-g_{\min }$ as $\epsilon \rightarrow 0$ for Instance 3 .

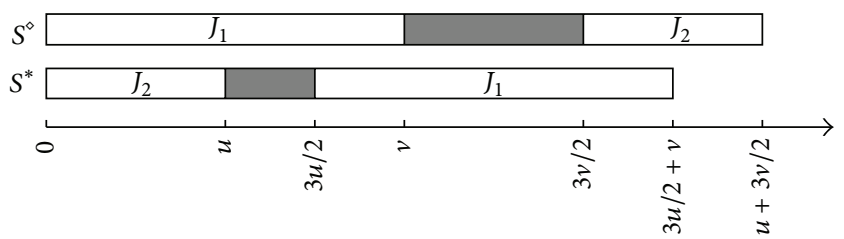

FIgURE 4: Schedules for Instance 4.

Case 4. $\lambda^{\diamond}>u$ and $\lambda^{*}>u$. Consider the following.

It is easy to see that in this case we have (16) and (21). And hence,

$$
L_{\max }^{\diamond} \leq L_{\max }^{*}+f(v)-f(u) .
$$

To see that this bound is tight, consider the following instance.

Instance 4. Consider $n=2, p_{1}=v, p_{2}=u, d_{2}=d_{1}+\epsilon$, and $f(x)=x / 2$, where $\epsilon \leq u / 4$.

It can be checked that for this instance $L_{\max }^{\diamond}=L_{2}^{\diamond}=u+$ $3 v / 2-\epsilon-d_{1}$ while $L_{\max }^{*}=L_{1}^{*}=3 u / 2+v-d_{1}$ (see Figure 4). Note that for this instance $f(u)=u / 2$ and $f(v)=v / 2$. So we have $L_{\max }^{\diamond} \rightarrow L_{\max }^{*}+f(v)-f(u)$ as $\epsilon \rightarrow 0$ for Instance 4 .

This completes the proof.

\section{Theorem 5.}

$$
\begin{aligned}
L_{\max }^{\diamond} \leq L_{\max }^{*}+\max \{ & g_{\max }-g_{\min }, g_{\max }-g(u), \\
& \left.f(v)-u-g_{\min }, f(v)-f(u)\right\}
\end{aligned}
$$

and the bound is tight.

Proof. If job $J_{j_{i} \diamond}$ is scheduled to be processed before the maintenance in $S^{\diamond}$, then by Theorem 2, we know that $S^{\diamond}$ is optimal, which implies that (24) is true.

If job $J_{j_{i}}$ is scheduled to be processed after the maintenance in $S^{\diamond}$, then according to Theorem 4 , we have (8), which implies that we also have (24).

To see that this bound is tight, consider Instance 3 that is presented in the proof of Theorem 4 again. It can be checked that for this instance $g_{\max }-g_{\min }=u, g_{\max }-g(u)=0, f(v)-$ $u-g_{\min }=u$, and $f(v)-f(u)=0$. And hence, for this instance, we have $\max \left\{g_{\max }-g_{\min }, g_{\max }-g(u), f(v)-u-g_{\min }, f(v)-\right.$ $f(u)\}=f(v)-u-g_{\min }$.

Recall that, for the same instance, we have $L_{\max }^{\diamond} \rightarrow$ $L_{\max }^{*}+f(v)-u-g_{\min }$ as $\epsilon \rightarrow 0$ according to the argument on Instance 3 in the proof of Theorem 4 . Therefore, for the same instance, we have $L_{\max }^{\diamond} \rightarrow L_{\max }^{*}+\max \left\{g_{\max }-g_{\min }, g_{\max }-\right.$ $\left.g(u), f(v)-u-g_{\min }, f(v)-f(u)\right\}$ as $\epsilon \rightarrow 0$.

This completes the proof.

\section{Conclusions}

In this paper, we considered a maximum lateness minimization scheduling problem on a single-machine with a mandatory maintenance whose duration is workload-dependent. The start time of the maintenance is restricted to a given 
time window. We showed that the problem is NP-hard and proposed an approximation algorithm for it.

Further research may focus on designing some sophisticated approximation algorithms with lower absolute bounds. It is also interesting to study such a maintenance model in some other machine settings, such as parallel machine, flow shop, and job shop.

\section{Conflict of Interests}

The authors declare that there is no conflict of interests regarding the publication of this paper.

\section{Acknowledgments}

This research was supported in part by the National Natural Science Foundation of China (71201022), the Natural Science Foundation of Jiangxi Province (20122BAB201010), and the Science Foundation of Education Committee of Jiangxi for Young Scholars (GJJ11144).

\section{References}

[1] J. R. Jackson, "Scheduling a production line to minimize maximum tardiness," Research Report 43, Management Science Research Project, University of California, Los Angeles, Calif, USA, 1955.

[2] R. Rudek, "The strong NP-hardness of the maximum lateness minimization scheduling problem with the processing-time based aging effect," Applied Mathematics and Computation, vol. 218, no. 11, pp. 6498-6510, 2012.

[3] Z. Jiang, F. Chen, and C. Wu, "Minimizing the maximum lateness in a single-machine scheduling problem with the normal time-dependent and job-dependent learning effect," Applied Mathematics and Computation, vol. 218, no. 18, pp. 9438-9441, 2012.

[4] N. Vakhania and F. Werner, "Minimizing maximum lateness of jobs with naturally bounded job data on a single machine in polynomial time," Theoretical Computer Science, vol. 501, pp. 7281, 2013.

[5] T. Chen, "An effective dispatching rule for bi-objective job scheduling in a wafer fabrication factory-considering the average cycle time and the maximum lateness," International Journal of Advanced Manufacturing Technology, vol. 67, no. 5-8, pp. 12811295, 2013.

[6] V. Sels and M. Vanhoucke, "A hybrid electromagnetism-like mechanism/tabu search procedure for the single machine scheduling problem with a maximum lateness objective," Computers \& Industrial Engineering, vol. 67, no. 1, pp. 44-55, 2014.

[7] Z. Liu and Y. K. Ro, "Rescheduling for machine disruption to minimize makespan and maximum lateness," Journal of Scheduling, vol. 17, no. 4, pp. 339-352, 2014.

[8] M. Ji, Y. He, and T. C. E. Cheng, "Single-machine scheduling with periodic maintenance to minimize makespan," Computers and Operations Research, vol. 34, no. 6, pp. 1764-1770, 2007.

[9] H.-T. Lee, D.-L. Yang, and S.-J. Yang, "Multi-machine scheduling with deterioration effects and maintenance activities for minimizing the total earliness and tardiness costs," International Journal of Advanced Manufacturing Technology, vol. 66, no. 1-4, pp. 547-554, 2013.
[10] D. Xu and D.-L. Yang, "Makespan minimization for two parallel machines scheduling with a periodic availability constraint: mathematical programming model, average-case analysis, and anomalies," Applied Mathematical Modelling, vol. 37, no. 14-15, pp. 7561-7567, 2013.

[11] D. Xu, A. Liu, and D. L. Yang, "Mathematical programming models for competitive two-agent single-machine scheduling with flexible periodic maintenance activities," Arabian Journal for Science and Engineering, vol. 39, no. 5, pp. 3715-3722, 2014.

[12] Y. Yin, D. Ye, and G. Zhang, "Single machine batch scheduling to minimize the sum of total flow time and batch delivery cost with an unavailability interval," Information Sciences, vol. 274, pp. 310-322, 2014.

[13] S.-J. Yang, C.-J. Hsu, and D.-L. Yang, "Single-machine scheduling and slack due-date assignment with aging effect and deteriorating maintenance," Optimization Letters, vol. 6, no. 8, pp. 1855-1873, 2012.

[14] T. C. E. Cheng, S. J. Yang, and D. L. Yang, "Common duewindow assignment and scheduling of linear time-dependent deteriorating jobs and a deteriorating maintenance activity," International Journal of Production Economics, vol. 135, no. 1, pp. 154-161, 2012.

[15] Y. Yin, W.-H. Wu, T. C. E. Cheng, and C.-C. Wu, "Due-date assignment and single-machine scheduling with generalised position-dependent deteriorating jobs and deteriorating multimaintenance activities," International Journal of Production Research, vol. 52, no. 8, pp. 2311-2326, 2014.

[16] W. Luo, L. Chen, and G. Zhang, "Approximation algorithms for scheduling with a variable machine maintenance," in Algorithmic Aspects in Information and Management, vol. 6124 of Lecture Notes in Computer Science, pp. 209-219, 2010.

[17] M. L. Pinedo, Scheduling: Theory, Algorithms, and Systems, Springer, New York, NY, USA, 4th edition, 2012.

[18] M. R. Garey and D. S. Johnson, Computers and Intractrability: A Guide to the Theory of NP-Completeness, Freeman, New York, NY, USA, 1979. 


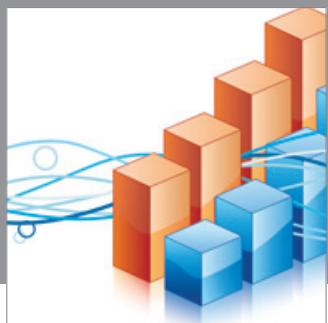

Advances in

Operations Research

mansans

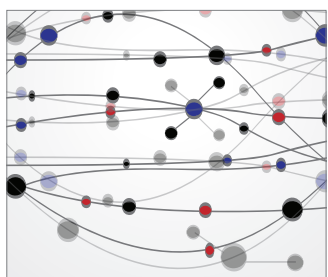

The Scientific World Journal
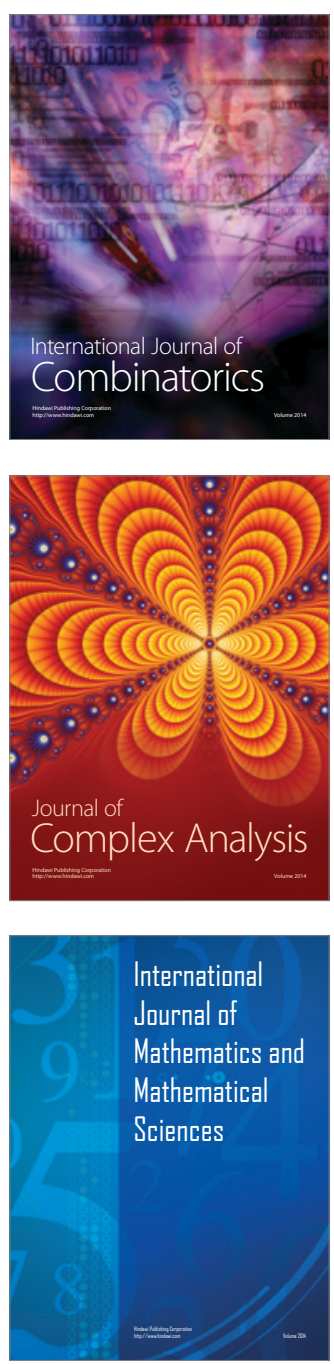
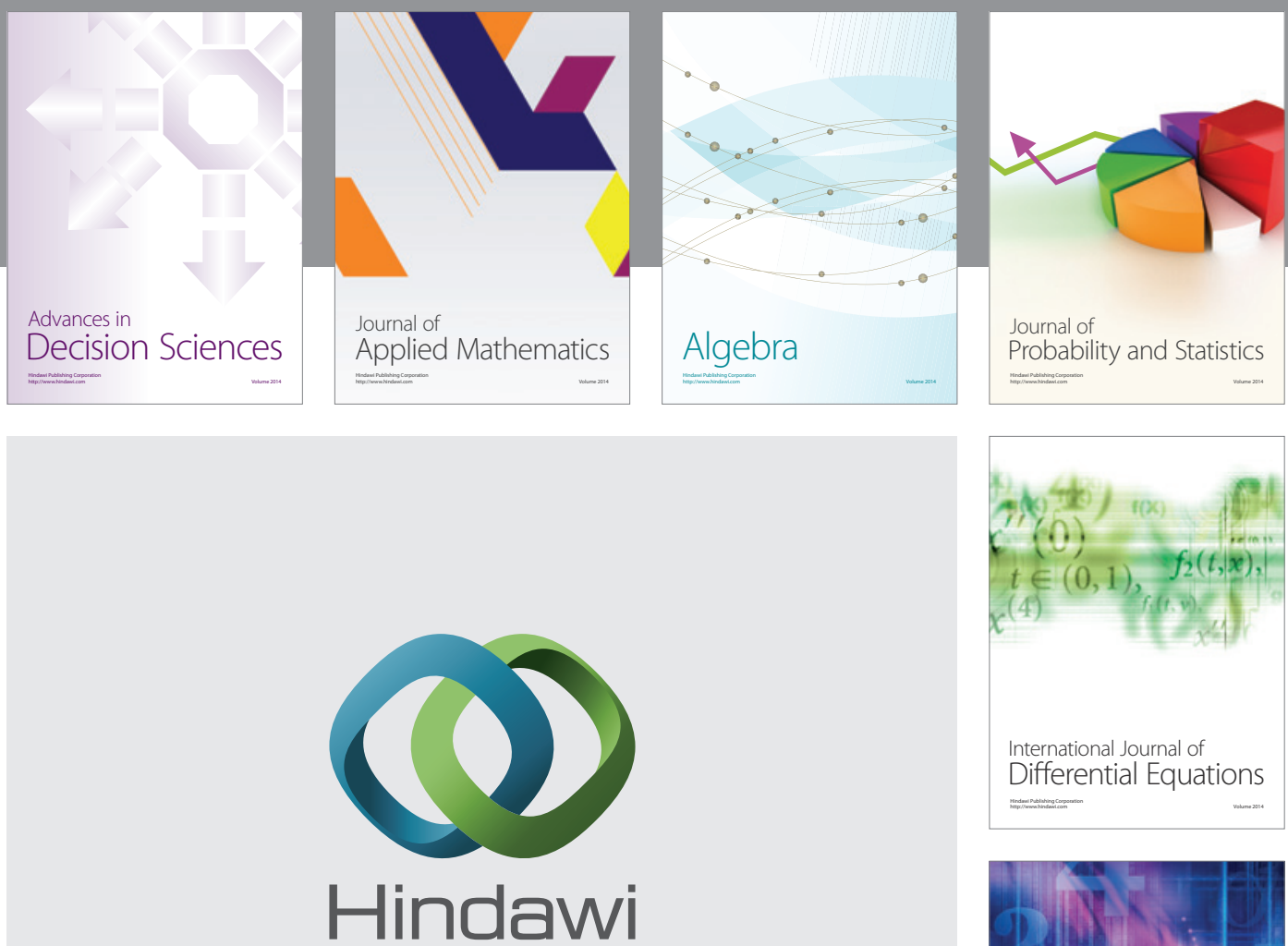

Submit your manuscripts at http://www.hindawi.com
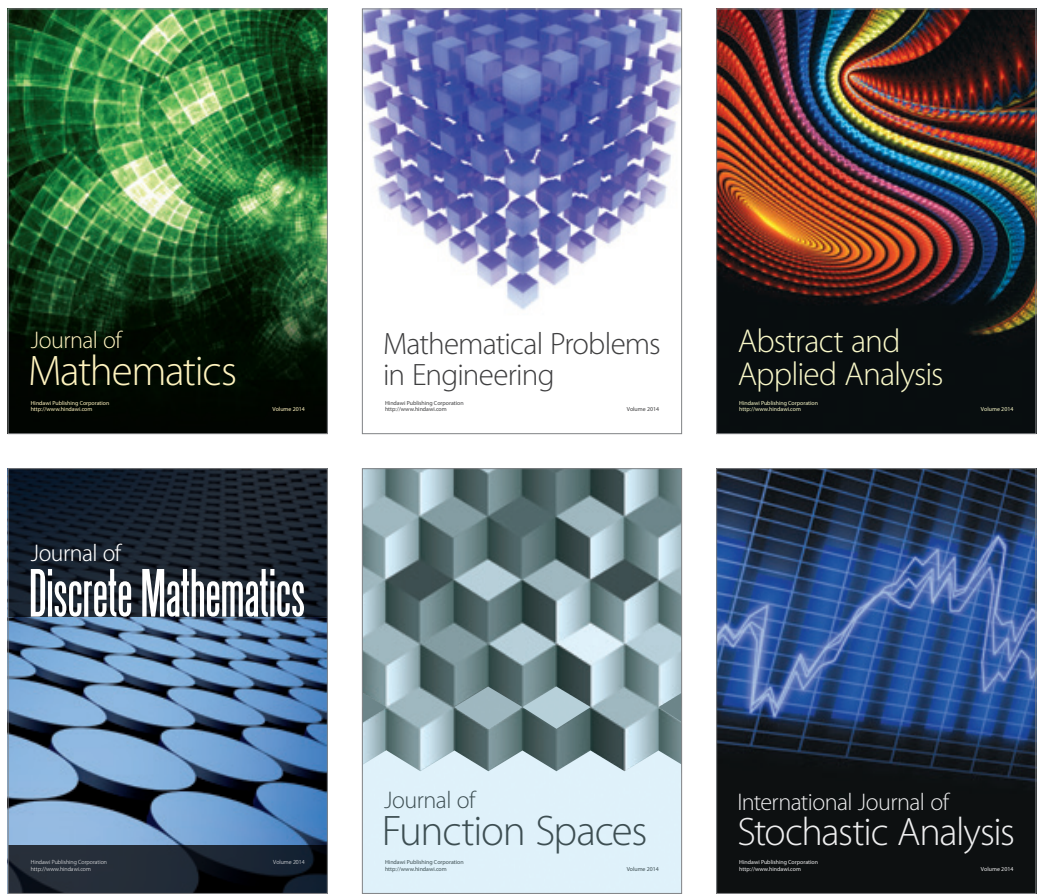

Journal of

Function Spaces

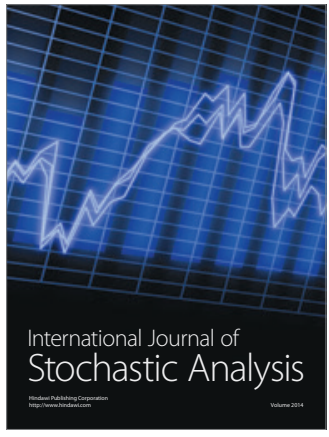

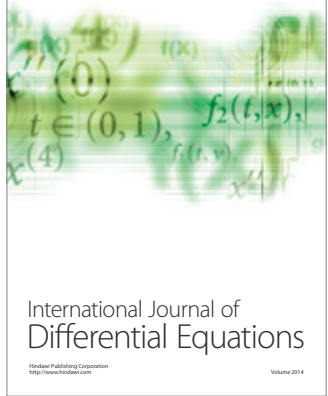
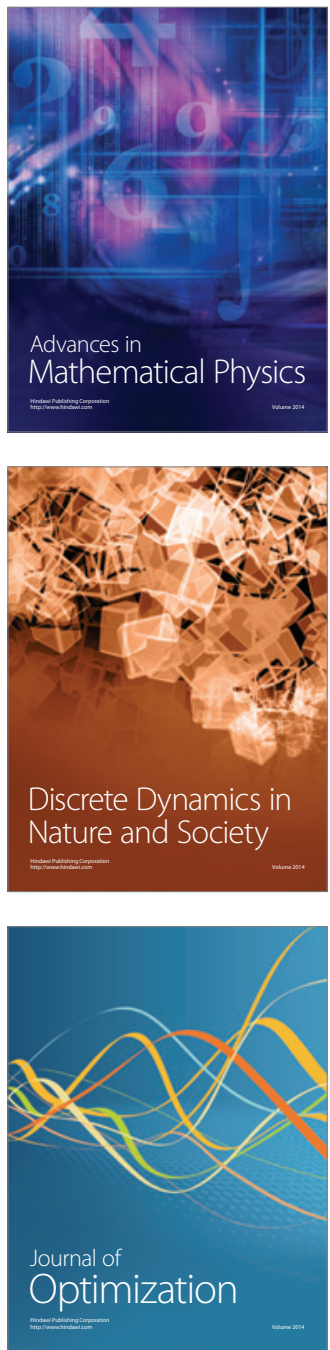